\title{
Asteroseismology of the PG 1159 star PG 0122+200^
}

J.-N. $\mathrm{Fu}^{1,2}$, G. Vauclair ${ }^{2}$, J.-E. Solheim ${ }^{3}$, M. Chevreton ${ }^{4}$, N. Dolez $^{2}$, M. S. O’Brien ${ }^{5}$, S.-L. Kim ${ }^{6}$, B.-G. Park ${ }^{6}$, G. Handler ${ }^{7}, 8$, R. Medupe ${ }^{8}$, M. Wood ${ }^{9}$, J. Gonzalez Perez ${ }^{10}$, O. Hashimoto ${ }^{11}$, K. Kinugasa $^{11}$, H. Taguchi ${ }^{11}$, E. Kambe ${ }^{11}$, J. Provencal ${ }^{12}$, S. Dreizler ${ }^{13,14}$, S. Schuh ${ }^{13,14}$, E. Leibowitz ${ }^{15}$, Y. Lipkin ${ }^{15}$, X.-B. Zhang ${ }^{16}$, M. Paparo ${ }^{17}$, B. Szeidl ${ }^{17}$, G. Virághalmy ${ }^{17}$, and D. Zsuffa ${ }^{17}$

1 Department of Astronomy, Beijing Normal University, Beijing, PR China

2 CNRS-UMR5572, Observatoire Midi-Pyrénées, University Paul Sabatier, Toulouse, France

Institute of Theoretical Astrophysics, University of Oslo, Oslo, Norway

${ }^{4}$ LESIA, Observatoire de Paris-Meudon, Meudon, France

5 Department of Astronomy, Yale University, New Haven, Connecticut, USA

6 Korea Astronomy and Space Science Institute, Daejon, Korea

7 Institute für Astronomie, Universität Wien, Wien, Austria

8 South African Astronomical Observatory, South Africa

9 Department of Physics and Space Sciences \& SARA Observatory, Florida Institute of Technology, Florida, USA

${ }^{10}$ IAC, La Laguna, Tenerife, Spain

11 Gunma Astrophysical Observatory, Japan

12 Mount Cuba Observatory and University of Delaware, Newark, Delaware, USA

13 Institut für Astrophysik, Friedrich-Hund-Platz 1, Göttingen, Germany

14 Institut für Astronomie und Astrophysik, Tübingen, Germany

15 Wise Observatory, Tel Aviv University, Tel Aviv, Israel

16 National Astronomical Observatories, Beijing, China

17 Konkoly Observatory, Budapest, Hungary

Received 23 August 2006 / Accepted 4 February 2007

\section{ABSTRACT}

Context. The variable pre-white dwarf PG 1159 stars (GW Vir) are $g$-mode non-radial pulsators. Asteroseismology puts strong constraints on their global parameters and internal structure. PG 0122+200 defines the red edge of the instability strip and its evolutionary timescale is predicted to be dominated by neutrino emission. Its study offers the opportunity to better understand the instability mechanism and to validate the physics of the neutrino production in dense plasma.

Aims. To achieve such a goal requires determining precisely its fundamental parameters. This is the goal of this paper.

Methods. We present new multi-site photometric observations obtained in 2001 and 2002. Together with previous data, they allow us to detect 23 frequencies, composed of 7 triplets and 2 single frequencies, which are used to constrain its internal structure and derive its fundamental parameters.

Results. All the observed frequencies correspond to $\ell=1 \mathrm{~g}$-modes. The period distribution shows a signature of mode trapping from which we constrain the He-rich envelope mass fraction to be $-6.0 \leq \log \left(q_{y}\right) \leq-5.3$. The comparison of the mode trapping amplitudes among GW Vir stars suggests that the mass-loss efficiency must decrease significantly below $T_{\text {eff }} \leq 140 \mathrm{kK}$. We measure an average period spacing of $22.9 \mathrm{~s}$ from which we derive a mass of $0.59 \pm 0.02 M_{\odot}$. From the triplets we measure a mean rotational splitting of $3.74 \mu \mathrm{Hz}$ and a rotational period of 1.55 days. We derive an upper limit to the magnetic field of $B \leq 4 \times 10^{3} \mathrm{G}$. The luminosity $\left(\log L / L_{\odot}=1.3 \pm 0.5\right)$ and the distance $\left(D=0.7_{-0.4}^{+1.0} \mathrm{kpc}\right)$ are only weakly constrained due to the large uncertainty on the spectroscopically derived surface gravity and the absence of a measured parallax.

Conclusions. From the asteroseismic mass, the ratio of the neutrino luminosity on the photon luminosity is $1.6 \pm 0.2$ confirming that the PG $0122+200$ evolutionary time scale should be dominated by neutrino cooling. A measurement of $\dot{P}$ for the largest amplitude untrapped modes should verify this prediction.

Key words. stars: evolution - stars: fundamental parameters - stars: white dwarfs - stars: oscillations - stars: individual: PG $0122+200$

\footnotetext{
* Based on data obtained at the Haute-Provence Observatory, INSU/CNRS, France; the Nordic Optical Telescope, La Palma, Spain; the Xinglong station of National Astronomical Observatories, China; the Bohyunsan Observatory, South-Korea; the Gunma Astrophysical Observatory, Japan; the Teide Observatory, Tenerife, Spain; the Calar Alto Observatory, Spain; the Kitt Peak National Observatory, Arizona, USA; the McDonald Observatory, Texas, USA; the South Africa Astronomical Observatory, South Africa; the Wise Observatory, Israel and the Piszkéstető Observatory, Hungary.
}

\section{Introduction}

The PG 1159 stars form an evolutionary link between the central stars of planetary nebulae and the white dwarf cooling sequence. A fraction of the PG 1159 stars does pulsate and constitutes the subgroup of the GW Vir type of variable stars. The asteroseismology of these variable stars provides invaluable insight on their internal structure and evolutionary status. Among the four known GW Vir stars, which have all been studied with the Whole Earth Telescope network (WET; Nather et al. 1990), 
PG $0122+200$ is the coolest one. Its atmospheric parameters $T_{\text {eff }}=80000 \mathrm{~K} \pm 4000 \mathrm{~K}, \log g=7.5 \pm 0.5$, and abundances $\left(\mathrm{C} / \mathrm{He}=0.3, \mathrm{C} / \mathrm{O}=3, \mathrm{~N} / \mathrm{He}=10^{-2}\right.$ by numbers $)$ are typical of the GW Vir stars (Dreizler \& Heber 1998). At this effective temperature, PG $0122+200$ presently defines the red edge of the GW Vir stars. O'Brien et al. (1998) have shown that at this phase of their evolution, the pre-white dwarfs cool predominantly through neutrino losses. The ratio of the neutrino luminosity over the photon luminosity could be as large as 2.5 in PG $0122+200$, if its mass is $0.66 M_{\odot}$. In PG 1159 035 , at $T_{\text {eff }}=140000 \mathrm{~K}$, this ratio is only of the order of 0.1 . This makes PG $0122+200$ of particular interest to study neutrino physics if one could measure its cooling rate from asteroseismology. However, the predicted neutrino luminosity depends on the total mass, which can be determined precisely in principle from asteroseismology.

Determining the mass of a non-radial g-mode pulsator relies on the period spacing and on the correct identification of the degree $\ell$ of the modes. After the discovery of its pulsations (Bond \& Grauer 1987), PG 0122+200 was observed in 1986 (O’Brien et al. 1996), in 1990 (Vauclair et al. 1995) and was the priority target of a WET campaign in 1996 (O’Brien et al. 1998). From the accumulated periods observed during these three campaigns, it was not possible to determine unambiguously its period spacing. Both $16 \mathrm{~s}$ and $21 \mathrm{~s}$ could fit almost equally well the observed period distribution. The amplitude variations which make the amplitude of some modes to fall below the detection limit sometimes favor the $16 \mathrm{~s}$ period spacing and sometimes favor the $21 \mathrm{~s}$ one. As a period spacing of about $21 \mathrm{~s}$ was measured unambiguously at that time in the two well studied GW Vir stars PG 1159035 (Winget et al. 1991) and PG 2131+066 (Kawaler et al. 1995; Reed et al. 2000) as well as in the pulsating central star of the planetary nebula NGC 1501 (Bond et al. 1996), this value was preferred for PG $0122+200$. This choice was reinforced later by the value of 21.6 s measured in RX J2117+3412 (Vauclair et al. 2002) and of $23.0 \mathrm{~s}$ measured in PG 1707+427 (Kawaler et al. 2004). We were however urged to determine the period spacing in PG $0122+200$ on firmer basis. The mass deduced from a $16 \mathrm{~s}$ period spacing is in excess of $0.75 M_{\odot}$ while it is $0.68 M_{\odot}$ for a $21 \mathrm{~s}$ period spacing, for $\ell=1$ modes. This difference translates in a substantial difference in the neutrino luminosity, and consequently on the cooling time scale. The ambiguity was partly left in favor of $21 \mathrm{~s}$ after the observational campaign of 1999 (Vauclair et al. 2001) which revealed some new pulsation modes. However, the derived period spacing was determined from only 4 complete triplets, after accumulating the data from 1986 to 1999. Since we were pursuing the observations of PG $0122+200$ aiming at a $\dot{P}$ measurement, we are now able to anchor its mass determination on firmer basis by adding new observational data obtained since 1999. In this paper, we present in Sect. 2 the new observations obtained in 2001 and 2002. In Sect. 3 we derived the main parameters of PG $0122+200$ from asteroseismology. Section 4 summarizes our results.

\section{Observations and data reduction}

PG 0122+200 (BB Psc) was observed in October and December 2001 and October 2002. The goal of having two observing campaigns in 2001 separated by only two months was to improve the precision of the frequency determinations. Tables 1 and 2 give the journals of the campaigns for October and December, 2001, respectively. In October 2001, we observed PG 0122+200 for 9.8 days, leading to a formal frequency resolution of $1.2 \mu \mathrm{Hz}$, with a duty cycle of $21 \%$. In December 2001, the star was
Table 1. Journal of observations: 2001 October.

\begin{tabular}{ccccc}
\hline \hline Run Name & Telescope & $\begin{array}{c}\text { Date } \\
\text { (UT) }\end{array}$ & $\begin{array}{c}\text { Start Time } \\
\text { (UTC) }\end{array}$ & Run Length (s) \\
\hline kj08B & NOT $2.5 \mathrm{~m}$ & 08 Oct. & $23: 20: 21$ & 4021 \\
kj09B & NOT $2.5 \mathrm{~m}$ & 09 Oct. & $01: 49: 29$ & 16582 \\
kj11B & NOT $2.5 \mathrm{~m}$ & 11 Oct. & $21: 19: 00$ & 27043 \\
kj12aB & NOT $2.5 \mathrm{~m}$ & 12 Oct. & $05: 24: 20$ & 3641 \\
kj12bB & NOT $2.5 \mathrm{~m}$ & 12 Oct. & $19: 50: 21$ & 35124 \\
pg1013xm: & BAO $2.16 \mathrm{~m}$ & 13 Oct. & $13: 48: 00$ & 23411 \\
pg1015xl: & BAO $2.16 \mathrm{~m}$ & 15 Oct. & $17: 06: 00$ & 7620 \\
pg1016xm: & BAO 2.16 m & 16 Oct. & $12: 02: 00$ & 20478 \\
jlp-0308: & McDonald 2.1 m & 17 Oct. & $08: 34: 00$ & 11340 \\
pg1018x1 & BAO 2.16 m & 18 Oct. & $11: 21: 00$ & 27840 \\
\hline
\end{tabular}

Table 2. Journal of observations: 2001 December.

\begin{tabular}{|c|c|c|c|c|}
\hline 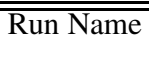 & $\overline{\text { Telescope }}$ & $\begin{array}{l}\text { Date } \\
\text { (UT) }\end{array}$ & $\begin{array}{c}\text { Start Time } \\
\text { (UTC) }\end{array}$ & "Run Length (s) \\
\hline calar-001 & Calar Alto $2.2 \mathrm{~m}$ & 07 Dec. & $18: 15: 49$ & 6295 \\
\hline bo-001 & BOAO $1.8 \mathrm{~m}$ & 08 Dec. & $09: 27: 27$ & 5921 \\
\hline wise-001: & Wise $1 \mathrm{~m}$ & 08 Dec. & $16: 06: 24$ & 4240 \\
\hline pi-001: & Piszkéstető $1 \mathrm{~m}$ & 08 Dec. & $17: 03: 13$ & 3994 \\
\hline mso-0051: & McDonald $2.1 \mathrm{~m}$ & 09 Dec. & $03: 16: 00$ & 19320 \\
\hline pi-002: & Piszkéstető 1 m & 09 Dec. & $16: 12: 54$ & 7665 \\
\hline wise-003: & Wise $1 \mathrm{~m}$ & 09 Dec. & $16: 34: 55$ & 3620 \\
\hline mso-0052 & McDonald $2.1 \mathrm{~m}$ & 10 Dec. & $01: 27: 00$ & 26690 \\
\hline mso-0054 & McDonald $2.1 \mathrm{~m}$ & 11 Dec. & $05: 55: 00$ & 9390 \\
\hline gh-0484 & SAAO $1 \mathrm{~m}$ & 11 Dec. & $18: 46: 40$ & 11850 \\
\hline mso-0055 & McDonald $2.1 \mathrm{~m}$ & 12 Dec. & 01:23:00 & 25890 \\
\hline gh-0486 & SAAO $1 \mathrm{~m}$ & 12 Dec. & $18: 31 ; 50$ & 10810 \\
\hline iac01 & Tenerife $0.85 \mathrm{~m}$ & 12 Dec. & $21: 20: 30$ & 7680 \\
\hline gh-0488 & SAAO $1 \mathrm{~m}$ & 13 Dec. & $18: 33: 20$ & 4760 \\
\hline iac02 & Tenerife $0.85 \mathrm{~m}$ & 13 Dec. & 21:10:00 & 3210 \\
\hline mso-0056 & McDonald $2.1 \mathrm{~m}$ & 14 Dec. & 01:20:00 & 24680 \\
\hline pi-003: & Piszkéstető $1 \mathrm{~m}$ & 14 Dec. & $16: 16: 10$ & 6242 \\
\hline gh-0490 & SAAO $1 \mathrm{~m}$ & 14 Dec. & $18: 26: 40$ & 6120 \\
\hline pg1215oh: & OHP $1.93 \mathrm{~m}$ & 15 Dec. & $17: 29: 00$ & 4415 \\
\hline gh-0492 & SAAO $1 \mathrm{~m}$ & 15 Dec. & 18:27:00 & 12690 \\
\hline pg1216oh: & OHP $1.93 \mathrm{~m}$ & 16 Dec. & $17: 28: 00$ & 20945 \\
\hline pg1217ok: & OHP $1.93 \mathrm{~m}$ & 17 Dec. & $17: 30: 00$ & 20007 \\
\hline gh-0494 & SAAO $1 \mathrm{~m}$ & 17 Dec. & $18: 29: 49$ & 1330 \\
\hline pg1218oh & OHP $1.93 \mathrm{~m}$ & 18 Dec. & $17: 27: 00$ & 23406 \\
\hline rm-0112: & SAAO $1 \mathrm{~m}$ & 18 Dec. & $18: 37: 22$ & 4210 \\
\hline gu-001 & Gunma $1.88 \mathrm{~m}$ & 19 Dec. & 08:34:42 & 1637 \\
\hline $\mathrm{rm}-0114 \mathrm{c}$ & SAAO $1 \mathrm{~m}$ & 19 Dec. & $18: 41: 15$ & 4390 \\
\hline gu-002 & Gunma $1.88 \mathrm{~m}$ & 20 Dec. & $08: 53: 11$ & 6000 \\
\hline $\mathrm{rm}-0116$ & SAAO $1 \mathrm{~m}$ & 20 Dec. & $18: 32: 14$ & 4720 \\
\hline $\mathrm{rm}-0118$ & $\mathrm{SAAO} 1 \mathrm{~m}$ & 21 Dec. & $18: 30: 10$ & 4520 \\
\hline gu-003 & Gunma $1.88 \mathrm{~m}$ & 22 Dec. & $10: 37: 11$ & 4470 \\
\hline $\mathrm{rm}-0120$ & $\mathrm{SAAO} 1 \mathrm{~m}$ & 22 Dec. & $18: 31: 40$ & 2780 \\
\hline sara127 & SARA $0.9 \mathrm{~m}$ & 23 Dec. & 01:13:00 & 13673 \\
\hline gu-004 & Gunma $1.88 \mathrm{~m}$ & 23 Dec. & 08:38:41 & 1290 \\
\hline $\mathrm{rm}-0122$ & SAAO $1 \mathrm{~m}$ & 23 Dec. & $18: 33: 32$ & 4310 \\
\hline gu-005 & Gunma $1.88 \mathrm{~m}$ & 24 Dec. & $08: 32: 11$ & 2070 \\
\hline
\end{tabular}

observed for a longer period of 16.6 days, leading to a formal frequency resolution of $0.7 \mu \mathrm{Hz}$, but with a lower duty cycle of $15 \%$. The star has been reobserved in October 2002. Table 3 gives the journal of the observations. With a campaign of 12.5 days, the achieved formal frequency resolution is $0.93 \mu \mathrm{Hz}$ and the duty cycle is $43.5 \%$.

Most of the data come from CCD-photometers which use a $30 \mathrm{~s}$ observing cycle. For the data obtained from multi-channel PMT- photometers, we summed the data points so as to have a similar sampling rate of $30 \mathrm{~s}$. Between 3 and 6 comparison stars and 2 sky background fields are currently used in CCDphotometry. For the PMT-photometers, one comparison star and 
Table 3. Journal of observations: 2002 October.

\begin{tabular}{ccccc}
\hline \hline Run Name & Telescope & $\begin{array}{c}\text { Date } \\
\text { (UT) }\end{array}$ & $\begin{array}{c}\text { Start Time } \\
\text { (UTC) }\end{array}$ & Run Length (s) \\
\hline pg1003oj: & OHP $1.93 \mathrm{~m}$ & 03 Oct. & $22: 29: 00$ & 10600 \\
korea4 & BOAO $1.8 \mathrm{~m}$ & 04 Oct. & $12: 12: 00$ & 26880 \\
pg1004xl: & BAO $2.16 \mathrm{~m}$ & 04 Oct. & $13: 01: 00$ & 10600 \\
pg1004oh: & OHP $1.93 \mathrm{~m}$ & 04 Oct. & $19: 41: 00$ & 30240 \\
pg1005xl: & BAO $2.16 \mathrm{~m}$ & 05 Oct. & $12: 56: 00$ & 7200 \\
pg1005xp: & BAO $2.16 \mathrm{~m}$ & 05 Oct. & $16: 26: 00$ & 14760 \\
pg1005oj: & OHP $1.93 \mathrm{~m}$ & 05 Oct. & $21: 26: 00$ & 23820 \\
pg1006xl: & BAO $2.16 \mathrm{~m}$ & 06 Oct. & $12: 45: 00$ & 22710 \\
pg1006oh: & OHP $1.93 \mathrm{~m}$ & 06 Oct. & $19: 29: 00$ & 29570 \\
pg1007xl & BAO $2.16 \mathrm{~m}$ & 07 Oct. & $12: 20: 00$ & 23120 \\
pg1007xm: & BAO $2.16 \mathrm{~m}$ & 07 Oct. & $19: 01: 00$ & 4620 \\
pg1007oh: & OHP $1.93 \mathrm{~m}$ & 07 Oct. & $21: 33: 00$ & 21870 \\
korea8 & BOAO $1.8 \mathrm{~m}$ & 08 Oct. & $11: 15: 00$ & 31560 \\
pg1008oh: & OHP $1.93 \mathrm{~m}$ & 08 Oct. & $20: 00: 00$ & 13170 \\
korea9 & BOAO $1.8 \mathrm{~m}$ & 09 Oct. & $11: 14: 00$ & 32400 \\
korea10 & BOAO $1.8 \mathrm{~m}$ & 10 Oct. & $11: 12: 00$ & 30660 \\
not101002 & NOT $2.5 \mathrm{~m}$ & 10 Oct. & $22: 04: 20$ & 28800 \\
not111002 & NOT $2.5 \mathrm{~m}$ & 11 Oct. & $21: 06: 20$ & 32640 \\
not121002 & NOT $2.5 \mathrm{~m}$ & 12 Oct. & $21: 10: 10$ & 33600 \\
not131002 & NOT $2.5 \mathrm{~m}$ & 13 Oct. & $21: 09: 20$ & 10300 \\
not141002 & NOT $2.5 \mathrm{~m}$ & 14 Oct. & $21: 36: 00$ & 31880 \\
not151002A & NOT $2.5 \mathrm{~m}$ & 15 Oct. & $21: 25: 40$ & 8500 \\
not151002B: & NOT $2.5 \mathrm{~m}$ & 16 Oct. & $00: 11: 00$ & 1480 \\
not151002C & NOT $2.5 \mathrm{~m}$ & 16 Oct. & $03: 38: 39$ & 10360 \\
\hline & & & &
\end{tabular}

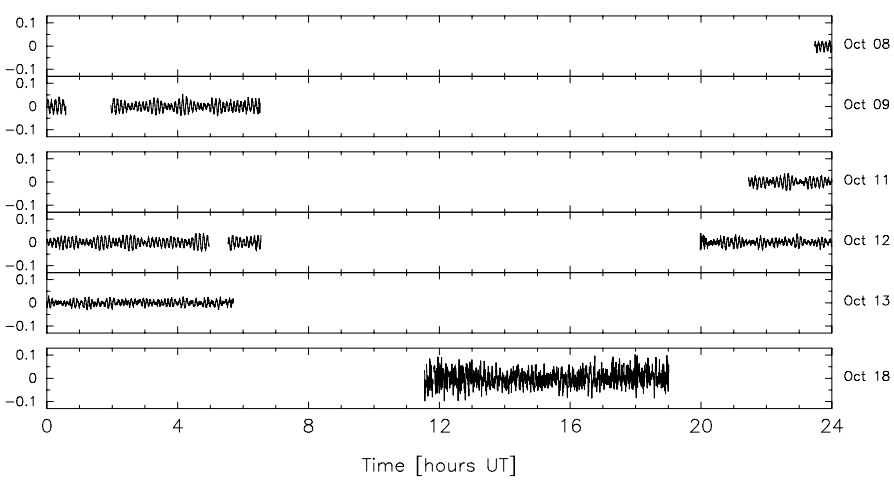

Fig. 1. Normalized light curve of the October 2001 campaign. Each panel covers a $24 \mathrm{~h}$ period; the date is indicated on the right side and the fractional amplitude on the left side. The $24 \mathrm{~h}$ periods when there was no data, or where we did not use the data (Oct. 10 and between Oct. 13 and Oct. 18) are not shown.

one sky background field are measured. Since our aim was to detect as many modes as possible in the power spectrum of the light curves, including small amplitude ones, we have been very conservative in selecting only the best data to construct the final light curves used for the frequency analysis. For this reason, some of the runs listed in Tables $1-3$ have not been included in the final light curves, either because the data were too poor due to bad observing conditions or technical problems, or were overlapping with better data obtained from the other runs, or were not of adequate signal/noise ratio. The runs which we did not use are indicated by colons. The duty cycles indicated above take into account only the data which have been used in the final reduction.

The differential light curves of the October 2001, December 2001 and October 2002 runs, normalized by subtracting to each light curve its average value, are shown in Figs. 1-3, respectively.

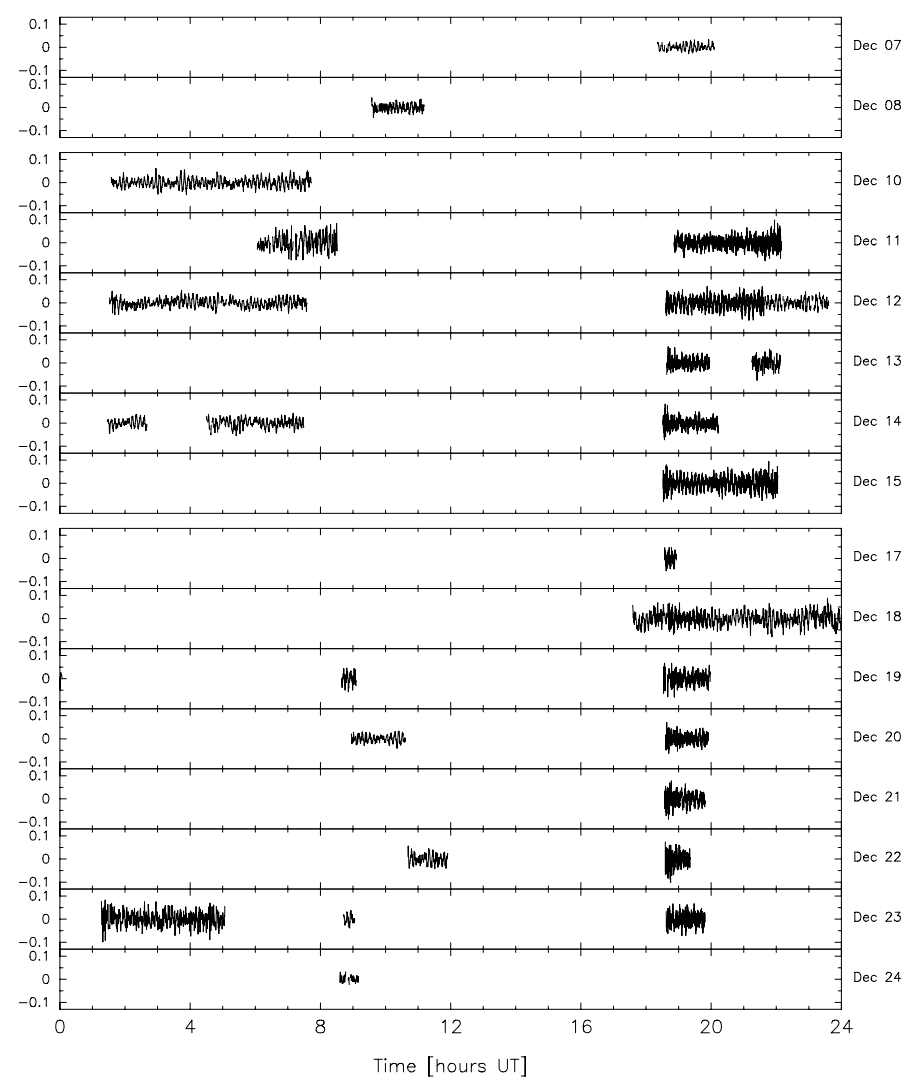

Fig. 2. Same as Fig. 1 for the December 2001 campaign. Dec. 9 and 16 where there was no usable data are not shown.

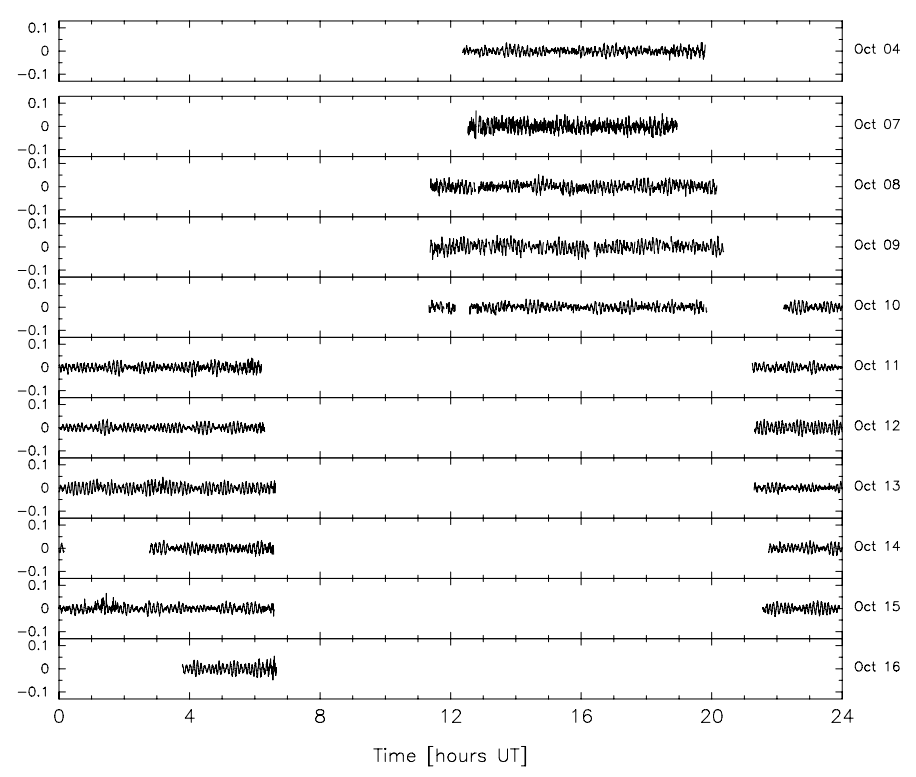

Fig. 3. Same as Fig. 1 for the October 2002 campaign. Oct. 5 and 6, where there was no usable data are not shown.

\section{Asteroseismology of PG $\mathbf{0 1 2 2 + 2 0 0}$}

\subsection{The power spectrum of the 2001 and 2002 campaigns}

We used the Period04 software (Lenz \& Breger 2005) to analyse the light curves and derive the frequencies, amplitudes and phases of the peaks in the Fourier transforms. Since the purpose of having two campaigns in 2001 separated by two months was to improve the frequency resolution and to get higher precision on the frequencies, we performed the Fourier transform 


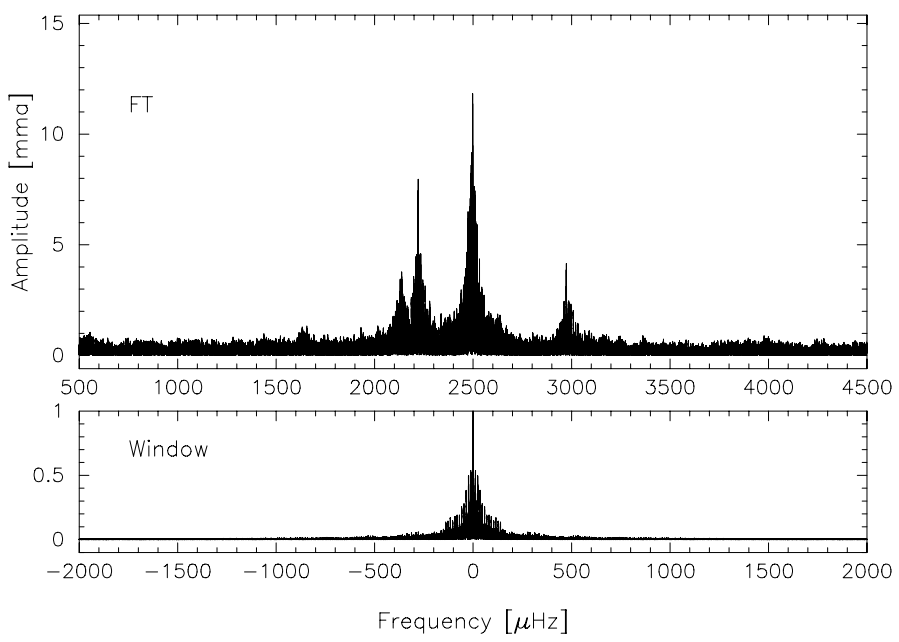

Fig. 4. The upper panel shows the amplitude spectrum of the combined light curve of October and December 2001; the amplitude, in units of milli-modulation amplitude (mma), is shown as a function of the frequency in $\mu \mathrm{Hz}$ in the frequency range $500-4500 \mu \mathrm{Hz}$; outside this frequency range no significant signal is detected. The lower panel is the corresponding window function on the same frequency scale.

of the combined light curves of the two runs. The resulting amplitude spectrum is shown in Fig. 4 on the frequency range between $500 \mu \mathrm{Hz}$ and $4500 \mu \mathrm{Hz}$. The average noise level is about $0.5 \mathrm{mma}$. The amplitude spectrum of a sinusoidal function sampled at the same rate as the data and having an amplitude equal to unity when there are data and equal to zero in the gaps (the window function) is also shown on the same scale. Figure 5 shows the same amplitude spectrum and window function on an enlarged frequency scale covering the range 2000-3200 $\mu \mathrm{Hz}$.

The amplitude spectrum of the October 2002 light curve is shown in Fig. 6 with the corresponding window function. For this run the noise level varies between 0.3 and $0.2 \mathrm{mma}$. An extended amplitude spectrum is shown in Fig. 7 covering the frequency range from $1400 \mu \mathrm{Hz}$ to $3200 \mu \mathrm{Hz}$ with the corresponding window function on the same scale.

For each of the peaks extracted by Period04, we computed the False Alarm Probability (FAP; Scargle 1982) for that peak to be due to noise. As can be seen from Figs. 4 and 6 , the noise level is almost constant in the frequency domain where signal is present. We used the average value of the noise in the frequency interval $500-4500 \mu \mathrm{Hz}$ to estimate the FAP for one peak to be due to noise in the frequency range from 0 to the Nyquist frequency. For the amplitude spectrum of the combined data of 2001, we estimate that any peak of signal over noise ratio $(S / N) \geq 4.30$ has a probability lower than $10^{-3}$ to be due to noise. This probability decreases to less than $10^{-4}$ for peaks with $S / N \geq 4.56$. For the 2002 data, the FAP for one peak to be due to noise is lower than $10^{-3}$ for $S / N \geq 4$. 1 . It is lower than for the 2001 combined data since there are a smaller number of independent points in the frequency spectrum. In the following, we adopt a conservative selection criterion and keep as significant frequencies only those with a: $F A P \leq 10^{-3}$.

The list of the significant frequencies, with their amplitude and phase, is given in Table 4, with their uncertainties. These uncertainties are the formal errors derived from the non-linear least-squares fits as performed by the Period04 software. We note that the combined 2001 runs allow us to determine the frequencies with an accuracy better by factor 2 to 8 compared to the 2002 run. This is at the expense of the noise since the duty cycle of the 2001 combined campaigns is much lower than the duty

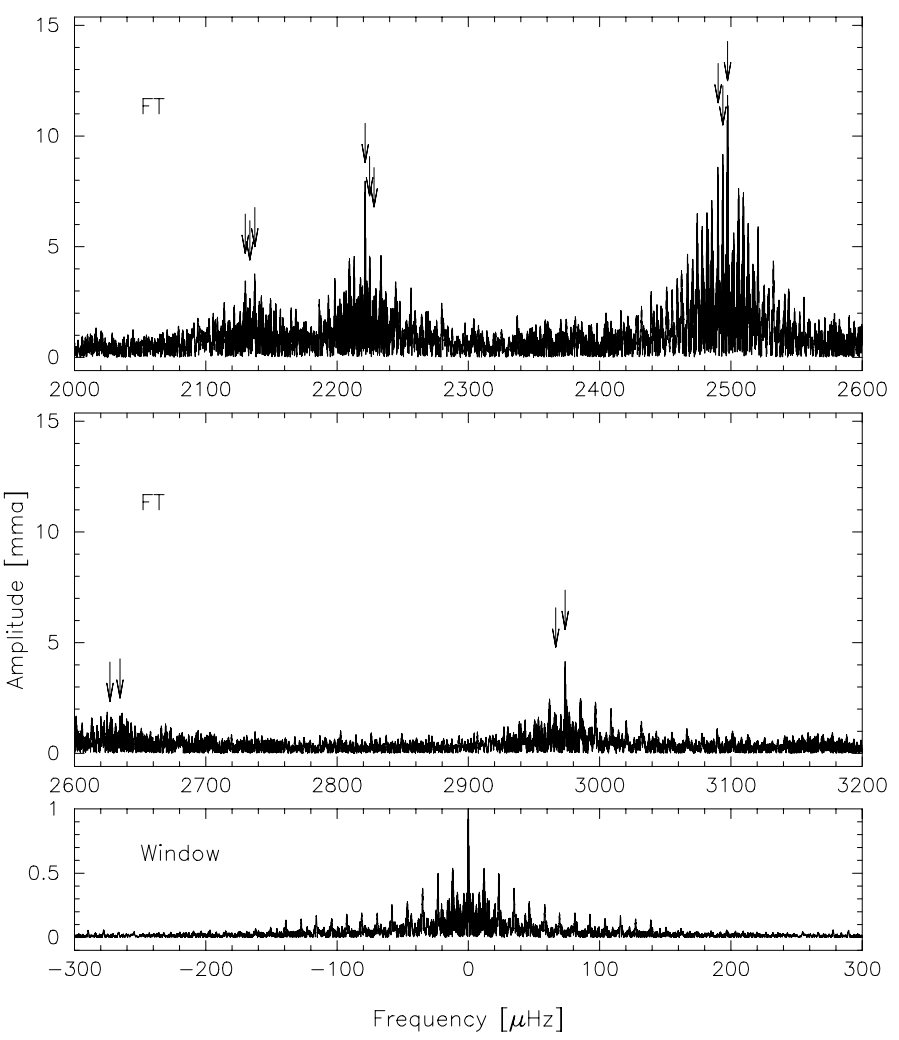

Fig. 5. Same as Fig. 4 on an extended scale. It shows the region between $2000 \mu \mathrm{Hz}$ and $3200 \mu \mathrm{Hz}$ where signal is detected. Each of the two upper panels covers a frequency range of $600 \mu \mathrm{Hz}$ between $2000 \mu \mathrm{Hz}$ and $2600 \mu \mathrm{Hz}$ and between $2600 \mu \mathrm{Hz}$ and $3200 \mu \mathrm{Hz}$ respectively. The arrows mark the multiplets identified after the pre-whitening process. The lower panel shows the window function on the same extended frequency scale.

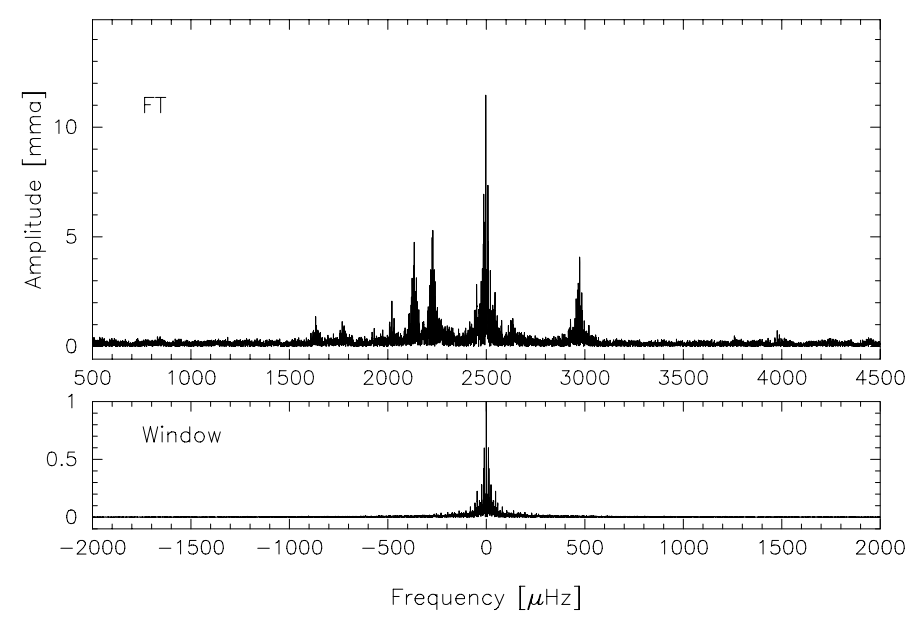

Fig. 6. Same as Fig. 4 for the light curve of October 2002.

cycle of the 2002 campaign. This is reflected in the smaller number of significant frequencies detected in 2001, since the smaller amplitude peaks are not selected, and in the larger uncertainty on the amplitude, by a factor of 2 . However, we checked that some of the frequencies significantly present in the 2002 data are also in the 2001 data below the detection limit. This is the case for the $2627 \mu \mathrm{Hz}$ and $2634 \mu \mathrm{Hz}$ peaks which are added in Table 4 for the 2001 data and marked with a colon. During these observing runs, the light curve was dominated by the $2497 \mu \mathrm{Hz}$ frequency. That was also the dominant frequency in all previous observing 
Table 4. Frequencies in PG0122+200.

\begin{tabular}{cccccccc}
\hline \hline $\begin{array}{c}\text { Frequency (2002) } \\
(\mu \mathrm{Hz})\end{array}$ & $\begin{array}{c}\sigma \\
(\mu \mathrm{Hz})\end{array}$ & $\begin{array}{c}A \pm 0.09 \\
(\mathrm{mma})\end{array}$ & $\phi$ & $\begin{array}{c}\text { Frequency }(2001) \\
(\mu \mathrm{Hz})\end{array}$ & $\begin{array}{c}\sigma \\
(\mu \mathrm{Hz})\end{array}$ & $\begin{array}{c}A \pm 0.19 \\
(\mathrm{mma})\end{array}$ & $\phi$ \\
\hline 1632.968 & 0.040 & 1.24 & 0.63 & & & & \\
1636.248 & 0.067 & 0.73 & 0.60 & & & & \\
1768.063 & 0.049 & 1.00 & 0.82 & & & & \\
1772.158 & 0.056 & 0.88 & 0.81 & & & & \\
1777.122 & 0.065 & 0.76 & 0.52 & & & & \\
1930.637 & 0.059 & 0.84 & 0.82 & & & & \\
2020.521 & 0.028 & 1.76 & 0.96 & & & & \\
2130.349 & 0.017 & 2.91 & 0.22 & 2130.089 & 0.005 & 3.26 & 0.33 \\
2133.749 & 0.013 & 3.87 & 0.08 & 2133.578 & 0.007 & 2.22 & 0.56 \\
2137.098 & 0.033 & 1.52 & 0.79 & 2137.316 & 0.004 & 3.81 & 0.71 \\
2221.488 & 0.016 & 3.04 & 0.01 & 2221.399 & 0.002 & 7.09 & 0.08 \\
2224.816 & 0.012 & 4.29 & 0.43 & 2224.760 & 0.004 & 3.90 & 0.96 \\
2228.157 & 0.010 & 4.82 & 0.33 & 2228.178 & 0.005 & 3.25 & 0.14 \\
2490.656 & 0.010 & 4.91 & 0.94 & 2490.243 & 0.003 & 5.60 & 0.71 \\
2493.797 & 0.011 & 4.39 & 0.53 & 2493.825 & 0.003 & 4.43 & 0.44 \\
2497.170 & 0.004 & 11.93 & 0.94 & 2497.412 & 0.002 & 9.87 & 0.02 \\
2627.265 & 0.034 & 1.44 & 0.42 & $2627.026:$ & 0.012 & 1.34 & 0.21 \\
2634.778 & 0.031 & 1.60 & 0.44 & $2634.649:$ & 0.009 & 1.80 & 0.41 \\
2966.750 & 0.019 & 2.64 & 0.03 & 2966.551 & 0.007 & 2.24 & 0.99 \\
2970.150 & 0.029 & 1.73 & 0.52 & & & & \\
2973.814 & 0.012 & 4.06 & 0.48 & 2973.731 & 0.003 & 4.44 & 0.90 \\
\hline
\end{tabular}

runs. Changes in the amplitude are noticeable on this one year timescale for the peaks at $2137 \mu \mathrm{Hz}$ and at $2221 \mu \mathrm{Hz}$.

\subsection{Period distribution and fine structure}

The amplitude spectrum of PG $0122+200$ derived from these new observations is very similar to those obtained during previous campaigns. There are a few changes in the amplitudes which allow us to complete the multiplets in many cases. This helps identifying the degree of the pulsation modes and constraining the model, as discussed below. The stability of the frequency spectrum gives us confidence in the possibility to derive the evolutionary time scale of PG $0122+200$ through the $\dot{P}$ measurement of at least one of its periods. It may be noticed also that there is no linear combination of frequencies detected, which suggests that all the significant peaks may be identified with real pulsation modes.

Power is present in a rather narrow frequency range, between $\approx 1630 \mu \mathrm{Hz}$ and $2970 \mu \mathrm{Hz}$, i.e. between $\approx 610 \mathrm{~s}$ and $336 \mathrm{~s}$. Examination of Table 4 reveals the presence of 7 multiplet structures, plus a couple of single frequencies, within this frequency range. In the following, we discuss the fine structure detected in the spectrum. When one frequency is obtained from the 2001 combined campaigns, we select that value of the frequency since the resolution of the combined runs of 2001 is better. Otherwise, we use the frequencies derived from the 2002 data or from an earlier multi-site campaign data when they allow us to complete a multiplet. In those last cases, the raw data of previous campaigns have been reanalyzed by using Period04 for homogeneity of the data reduction.

The two frequencies at $1632.9 \mu \mathrm{Hz}$ and $1636.2 \mu \mathrm{Hz}$ seen in 2002 are two components of a triplet whose third component is present at $1640.3 \mu \mathrm{Hz}$ in the 1999 data (Vauclair et al. 2001) and at $1640.5 \mu \mathrm{Hz}$ in the 1996 data (O'Brien et al. 1998). The $m=0$ component of this triplet was predicted at $1636.52 \mu \mathrm{Hz}$ from the frequencies observed by Vauclair et al. (2001) for the presumably $m= \pm 1$ components. It is detected here for the first time at $1636.248 \mu \mathrm{Hz}$.
The three frequencies around $1772 \mu \mathrm{Hz}$ form a complete triplet. The one day alias of its retrograde component may have been present in the 1999 data set (Vauclair et al. 2001) and in the 1986 single site data (O'Brien et al. 1996).

The next two frequencies at $1930.6 \mu \mathrm{Hz}$ and $2020.5 \mu \mathrm{Hz}$ are single frequencies. We will check later whether the proposed period spacing fits the period of these two modes.

The three frequencies around $2133.6 \mu \mathrm{Hz}$ form a complete triplet seen in both 2001 and 2002 campaigns. Only one or two components were detected in previous runs.

The three frequencies around $2224.7 \mu \mathrm{Hz}$ also form a complete triplet seen in all the multi-site campaigns.

The next three frequencies around $2493.8 \mu \mathrm{Hz}$ form a complete triplet which was always present in the multi-site campaigns. It contains the largest amplitude mode at $2497.4 \mu \mathrm{Hz}$, which noticeably is the prograde $(m=+1)$ component.

The next two frequencies at $2627.0 \mu \mathrm{Hz}$ and $2634.6 \mu \mathrm{Hz}$ are two components of a triplet whose third component was present alone in the 1996 data at $2630.8 \mu \mathrm{Hz}$ (O'Brien et al. 1998).

Finally, the last three frequencies around $2970.1 \mu \mathrm{Hz}$ form a complete triplet of which only one or two components had been seen before.

As a summary, combining the new data of 2001 and 2002 with some previous multi-site data we are able to identify 7 triplets and 2 single modes in the frequency distribution of PG $0122+200$. They are listed in Table 5.

\subsection{Rotational splitting and rotation rate}

Since all the observed multiplets are triplets, we consider that they correspond to $\ell=1$ modes split by rotation. Table 5 lists the frequency differences between the rotationally split components and the central component (Col. 3) for the 7 triplets. The averaged value of the frequency spacings is $3.74 \mu \mathrm{Hz}$. To the first order in the rotation angular frequency $\Omega$, the frequencies in the rotating case $\sigma_{k, l, m}$ are related to the frequencies in the non-rotating case $\sigma_{k, l}$ by:

$\sigma_{k, l, m}=\sigma_{k, l}+m \times\left(1-C_{k, l}\right) \Omega$. 


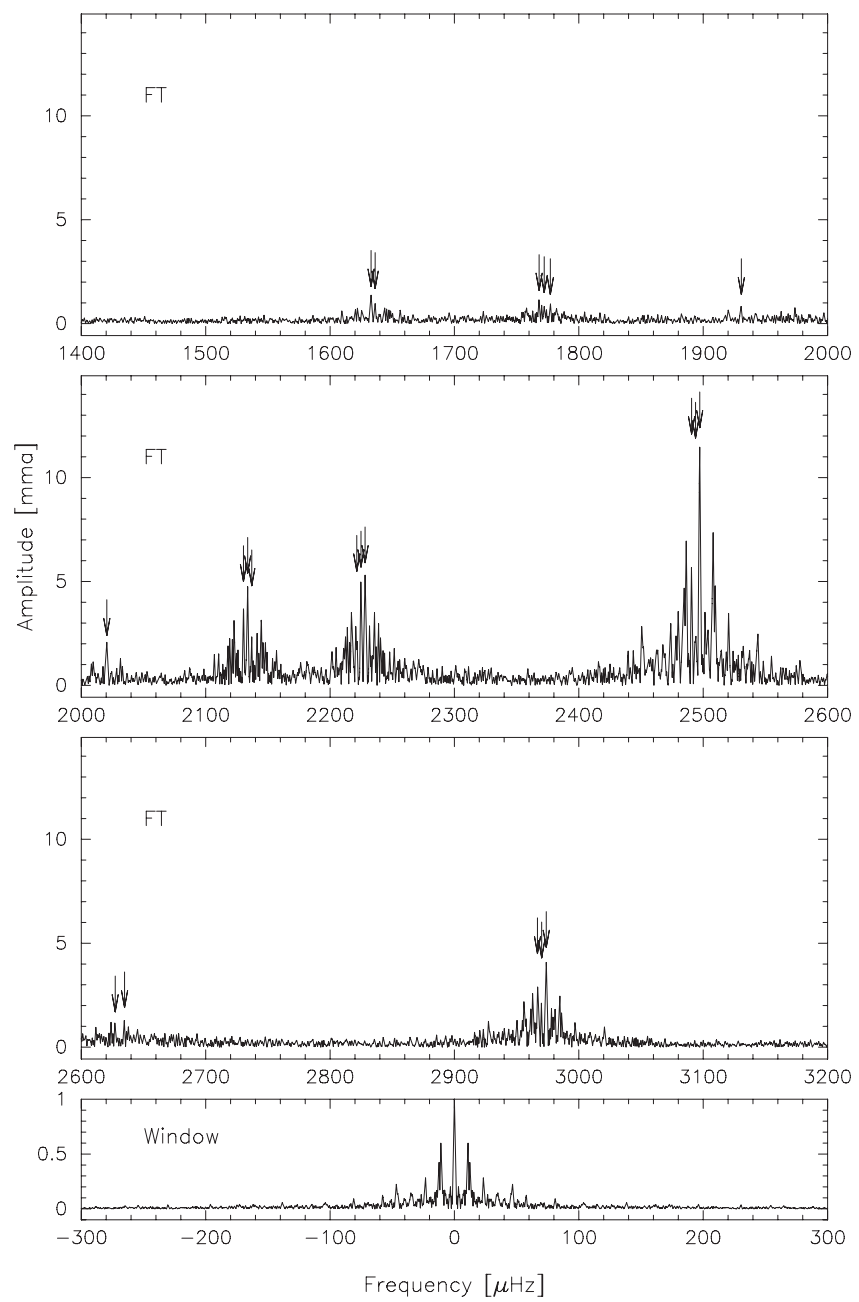

Fig. 7. This shows the same amplitude spectrum as Fig. 6 on an extended frequency scale. Each of the three upper panels shows a $600 \mu \mathrm{Hz}$ frequency range, between $1400 \mu \mathrm{Hz}$ and $2000 \mu \mathrm{Hz}, 2000 \mu \mathrm{Hz}$ and $2600 \mu \mathrm{Hz}, 2600 \mu \mathrm{Hz}$ and $3200 \mu \mathrm{Hz}$ respectively. The arrows mark the frequencies identified after the pre-whitening process. The lower panel shows the window function on the same frequency scale.

with $C_{k, l}=1 / \ell(\ell+1)$ in the asymptotic regime (Brickhill 1975). Assuming that it is the case of the pulsations observed in PG $0122+200$, we derive an average rotation period of 1.55 days.

This rotation rate lies within the range of rotation periods derived for the other hot pre-white dwarf pulsators, including the variable central stars of the planetary nebulae NGC 1510 (1.17 days; Bond et al. 1996) and RX J2117+3412 (1.16 days; Vauclair et al. 2002). For the three other GW Vir stars the rotation periods derived from asteroseismology are 1.38 days for PG 1159-035 (Winget et al. 1991), 0.21 days for PG 2131+066 (Kawaler et al. 1995) and 0.65 days or 1.30 days for PG 1707+427 (in this case only one doublet is seen, which could correspond to $\delta m=1$ or $\delta m=2$; Kawaler et al. 2004).

\subsection{Limit on magnetic field}

Considering the frequency spacing between the $m=0$ components of the triplets and the $m= \pm 1$ components, we find that the prograde splittings $(m=+1)$ are slightly larger, with an average splitting of $3.87 \pm 0.2 \mu \mathrm{Hz}$, than the retrograde $(m=-1)$ splittings which is only $3.61 \pm 0.11 \mu \mathrm{Hz}$ in average. The asymmetry of $0.26 \mu \mathrm{Hz}$ is marginally significant $(1 \times \sigma)$. It is however
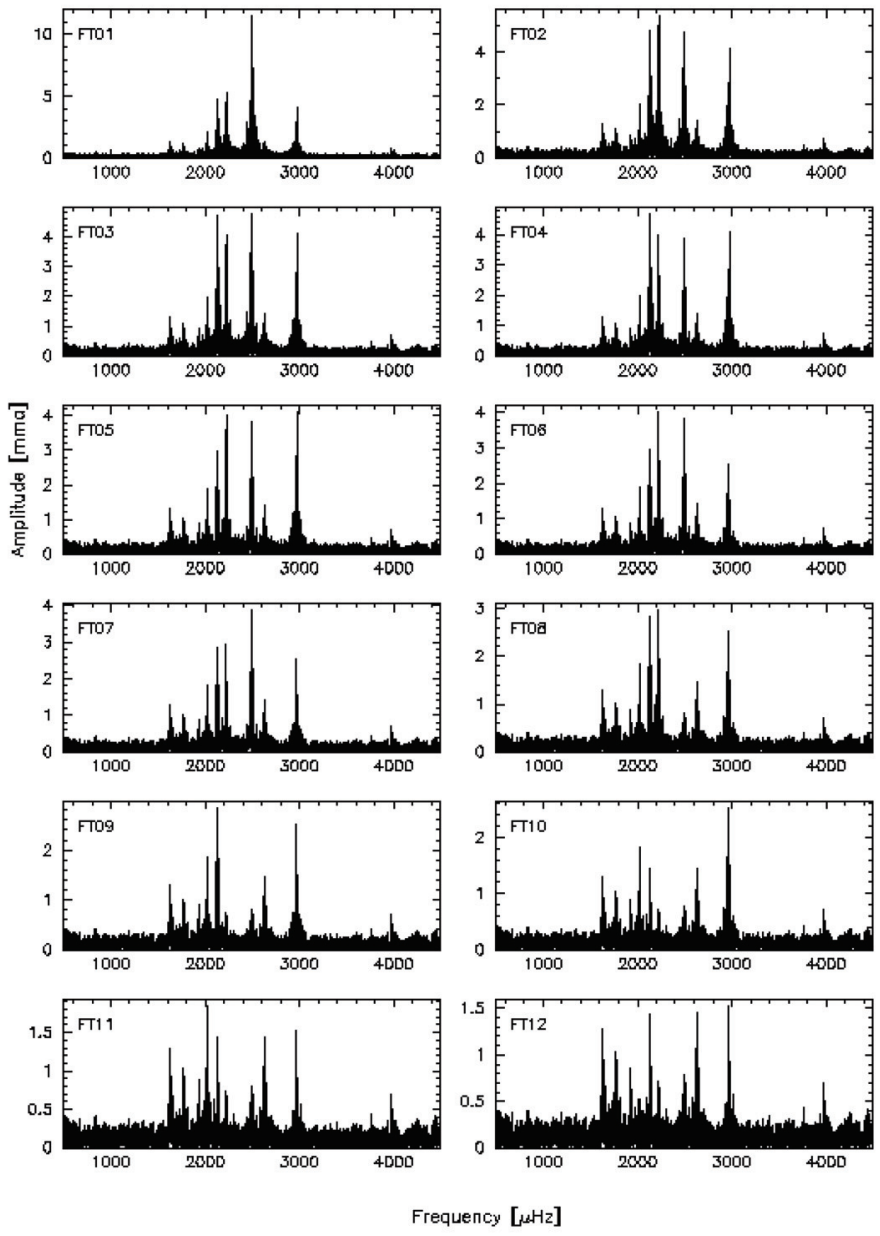

Fig. 8. Illustration of the prewhitening process on the October 2002 power spectrum. Only the twelwe first iterations of the prewhitening are shown. The first panel (FT01) is the global Fourier transform. Panel FT02 shows the Fourier transform of the light curve after subtracting the $2497.1 \mu \mathrm{Hz}$ frequency. The next panel shows the Fourier transform of the light curve after the additional subtraction of the $2228.1 \mu \mathrm{Hz}$, and so on for the subsequent panels. The process is stopped when no additional significant frequency is selected, according to the chosen detection limit. It the present case, the prewhitening process was stopped after 20 steps. The triplet centered on $2493.9 \mu \mathrm{Hz}$ is recoverd after the 8th iteration, the triplet centered on $2224.8 \mu \mathrm{Hz}$ after the 9th iteration. The corresponding peaks are identified with arrows in Fig. 7.

$4 \times \sigma$ of the worst formal error in the frequency determinations listed in Table 4 (for the mode at $1636.248 \mu \mathrm{Hz}$ ). If one interprets this frequency splitting difference as due to the effect of a weak magnetic field, we can evaluate what should be the strength of the field, following the approach of Jones et al. (1989). They note that the splitting due to a weak magnetic field as a function of the unperturbed periods does not vary by more than a factor of 3 for the models they have considered for PG 1159, DBV and DAV typical $T_{\text {eff }}$ and for $\ell$ from 1 to 3 . Accordingly, we based our estimate of the magnetic field on their figure 1 which is relevant to the DBV model for $\ell=1$ modes. At the shortest period observed in PG $0122+200$ (336 s), the frequency asymmetry could be explained by a magnetic field of $\approx 4 \times 10^{3} \mathrm{G}$. The constraint on the field could be stronger for the longest period $(610 \mathrm{~s})$ but, as mentionned by Jones et al. (1989), their working hypothesis may not be valid for long periods since the predicted frequency shifts correspond to period differences of the same order as the unperturbed periods, which disagrees with their assumptions. As 
Table 5. Period distribution in PG $0122+200$. The values indicated in italic are taken from the previous multi-site campaigns of 1999 for the $1640.30 \mu \mathrm{Hz}$ frequency (Vauclair et al. 2001) and of 1996 for the $2630.89 \mu \mathrm{Hz}$ frequency (O’Brien et al. 1998).

\begin{tabular}{|c|c|c|c|c|}
\hline $\begin{array}{c}\text { Frequency } \\
(\mu \mathrm{Hz})\end{array}$ & $\begin{array}{c}\text { Period } \\
\text { (s) }\end{array}$ & $\begin{array}{c}\delta f \\
(\mu \mathrm{Hz})\end{array}$ & $\overline{\overline{\Delta k}}$ & $\bar{~} \bar{m}$ \\
\hline 1632.97 & 612.38 & & & -1 \\
\hline 1636.25 & 611.15 & $\begin{array}{l}3.28 \\
4.05\end{array}$ & +9 & 0 \\
\hline 1640.30 & 609.64 & & & +1 \\
\hline 1768.06 & 565.59 & & & -1 \\
\hline 1772.16 & 564.28 & 4.10 & +7 & 0 \\
\hline 1777.12 & 562.70 & & & +1 \\
\hline 1930.64 & 517.96 & & +5 & 0 ? \\
\hline 2020.52 & 494.92 & & +4 & 0 ? \\
\hline 2130.09 & 469.46 & & & -1 \\
\hline 2133.58 & 468.69 & $\begin{array}{l}3.49 \\
3.73\end{array}$ & +3 & 0 \\
\hline 2137.31 & 467.87 & & & +1 \\
\hline 2221.40 & 450.16 & & & -1 \\
\hline 2224.76 & 449.48 & $\begin{array}{l}3.36 \\
3.42\end{array}$ & +2 & 0 \\
\hline 2228.18 & 448.79 & & & +1 \\
\hline 2490.24 & 401.56 & & & -1 \\
\hline 2493.82 & 400.99 & $\begin{array}{l}3.58 \\
3.59\end{array}$ & 0 & 0 \\
\hline 2497.41 & 400.41 & & & +1 \\
\hline 2627.02 & 380.66 & & & -1 \\
\hline 2630.89 & 380.10 & $\begin{array}{l}3.87 \\
3.76\end{array}$ & -1 & 0 \\
\hline 2634.65 & 379.55 & & & +1 \\
\hline 2966.55 & 337.09 & & & -1 \\
\hline 2970.15 & 336.68 & .60 & -3 & 0 \\
\hline 2973.73 & 336.28 & & & +1 \\
\hline
\end{tabular}

a conclusion, if the observed frequency asymmetry observed in the triplets components is real and can be attributed to the effect of a magnetic field, the upper limit of that field should be a few $10^{3} \mathrm{G}$. A stronger magnetic field would produce a frequency shift larger than the suspected $1 \times \sigma$ asymmetry. This value is comparable to the upper limit of $6 \times 10^{3} \mathrm{G}$ for the strength of the magnetic field in PG 1159-035 (Winget et al. 1991).

\subsection{Average period spacing}

The triplets observed in PG $0122+200$ are $\ell=1$ modes. The separation between modes of consecutive order should be equal to the period spacing, or close to its value to account for possible mode trapping. If the observed periods do not correspond to consecutive order modes, their period separation should be approximately a multiple of the period spacing. Examining the periods listed in Table 5, we see that the successive triplets are separated by $46.87 \mathrm{~s}, 95.59 \mathrm{~s}, 19.21 \mathrm{~s}, 48.49 \mathrm{~s}, 20.89 \mathrm{~s}$ and $43.42 \mathrm{~s}$ respectively. Since the triplets separated by only $19.21 \mathrm{~s}$ and $20.89 \mathrm{~s}$ have to be consecutive orders, this leads to the conclusion that the period spacing has to be $\approx 20 \mathrm{~s}$. We then observe that the various period intervals between the non consecutive modes are: $46.87 \mathrm{~s}=2 \times 23.43 \mathrm{~s}, 95.59 \mathrm{~s}=4 \times 23.89 \mathrm{~s}, 48.49 \mathrm{~s}=2 \times 24.24 \mathrm{~s}$ and $43.42 \mathrm{~s}=2 \times 21.71 \mathrm{~s}$. It suggests that one mode is missing in the interval of $46.87 \mathrm{~s}$ separating the first two triplets, other modes are missing between the triplet centered on $564.28 \mathrm{~s}$ and the single mode at $517.96 \mathrm{~s}$, between the triplets centered at $449.48 \mathrm{~s}$ and at $400.99 \mathrm{~s}$ and between the triplets at $380.10 \mathrm{~s}$ and at $336.68 \mathrm{~s}$. The total range of period, from $611.15 \mathrm{~s}$ to $336.68 \mathrm{~s}$, is filled by $\ell=1$ modes which, taking the missing modes into account, covers 13 orders. We deduce an average period spacing $\Delta P=22.9 \mathrm{~s}$.

To confirm our hypothesized period spacing in a more objective way, we applied three different methods to the distribution of periods given in Table 5. We used first the method proposed by Handler et al. (1997) to find regular spacings in data sets. The method consists in computing the spectral window function of a given data set. If a periodic gap is present between most of the data values, a maximum in the spectral window function of the data set will occur at the frequency of this gap, just as single site data that contain daily interruptions will result in a spectral window with a maximum at the one cycle per day alias, and with weaker maxima at its harmonics. We apply next the Kolmogorov-Smirnov test (K-S) as proposed by Kawaler(1988) and then the inverse variance test (IV) proposed by O'Donoghue (1994). The determination of the period spacing in PG $0122+200$ has long been ambiguous. We discuss here in details how the ambiguity between the earlier values of $16 \mathrm{~s}$ and 21 s occured. In a first step we applied each of the three methods to the seven $m=0$ mode periods and in a second step to these seven modes plus the two singlets at 495 and $518 \mathrm{~s}$.

The results are shown in Figs. 9-11 for the Handler's method, the K-S test and the IV test respectively. When applied only to the central modes of the seven multiplets, as shown in the upper panels for each figure, each of those three methods shows that there could be various possibilities for period spacings present: $22.9,16.0$ and $9.5 \mathrm{~s}$. In previous attempts to estimate the period spacing, either $21 \mathrm{~s}$ or $16 \mathrm{~s}$ was found depending on which modes were present in the data sets analyzed. It is why both values have been discussed earlier in the literature. When the two singlets are added, the ambiguity disappears as shown on the lower panel of the three figures. Now the $22.9 \mathrm{~s}$ period spacing clearly dominates, and we can reject the possibility of $16.0 \mathrm{~s}$ period spacing. We are thus confident that the modes we list in Table 5 are all $\ell=1$, and that they have an average spacing of $22.9 \mathrm{~s}$.

Figure 12 shows the linear least-squares fit of the seven $m=$ 0 modes. It confirms the $\Delta P=22.9 \mathrm{~s}$ period spacing. The two single modes for which one does not know the $m$ value fits nicely the period distribution with that value of the period spacing.

Previous estimates of the period spacing in PG $0122+200$ were based on a smaller number of well identified $m=0$ modes. In the WET data described in O'Brien et al. (1998) only one triplet was clearly identified and the $\Delta P$ was determined from this triplet, two more triplets observed earlier by Vauclair et al. (1995) and single periods of unknown $m$ value. Further data, described in Vauclair et al. (2001), allowed one to include one more triplet in the $\Delta P$ estimate. Here, we use a total number of seven triplets and two singlets. It is worth noting that even the use of the seven triplets may not have been sufficient to derive the period spacing. However, the addition of the two small amplitude singlets is important in fixing the period spacing unambiguously. These two modes at $1930 \mu \mathrm{Hz}$ and $2020 \mu \mathrm{Hz}$ have a $S / N$ ratio of 4.2 and 5.9 respectively in the amplitude Fourier spectrum of our 2002 data, while our detection limit was $S / N=4.1$ for a $F A P$ 

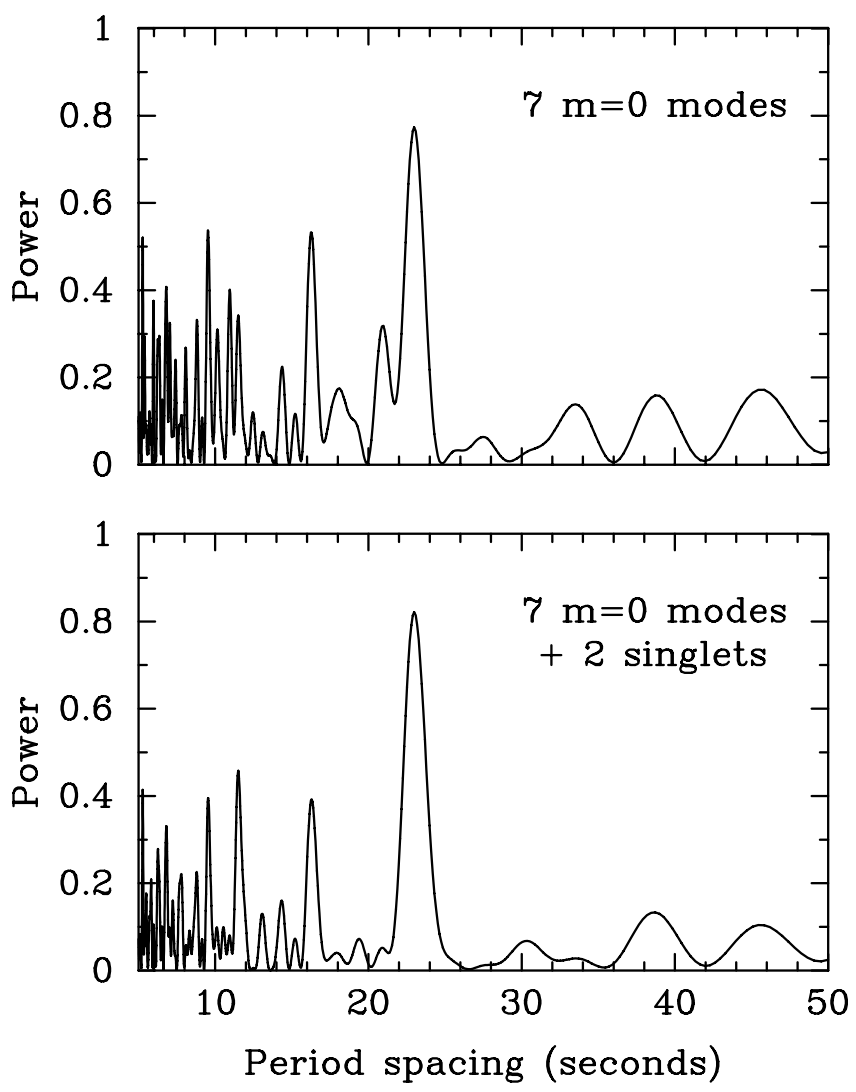

Fig. 9. Period spacing from Handler's method. Upper panel: the spectral window of the seven $m=0$ mode periods. Lower panel: the same, but with the two single modes at 495 and 518 s included.

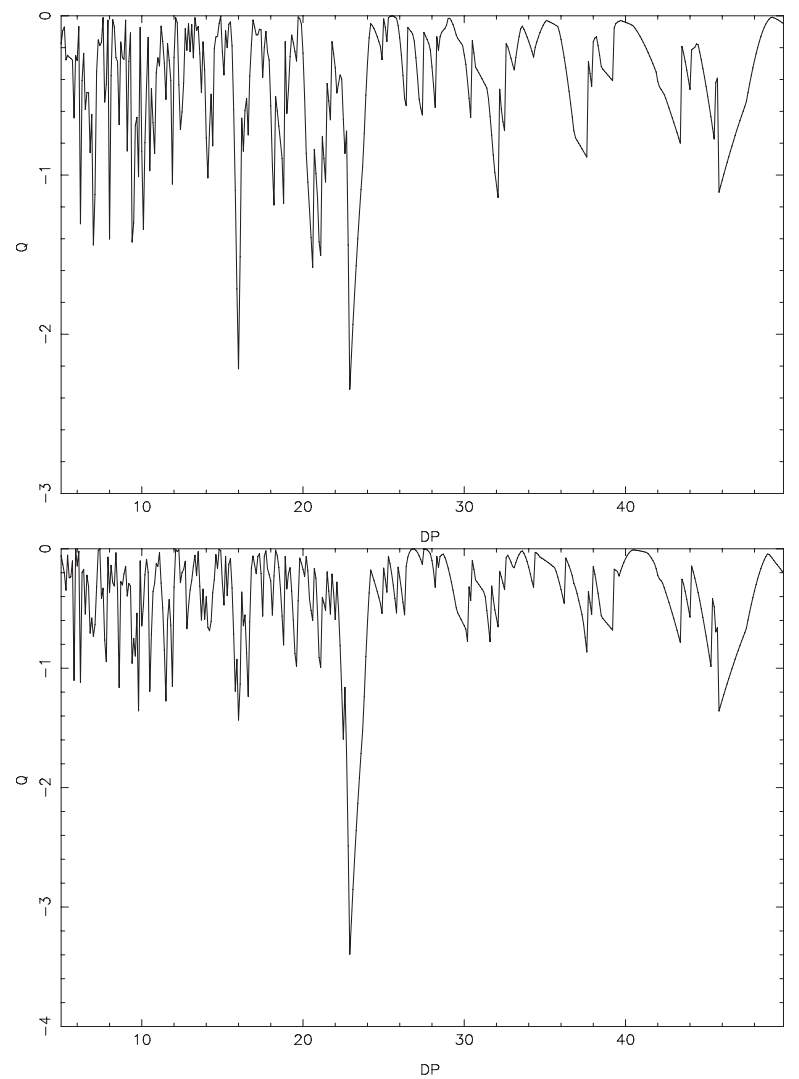

Fig. 10. Same as Fig. 9 from Kolmogorov-Smirnov test. Upper panel: the K-S test of the seven $m=0$ mode periods. Lower panel: the same, but with the two single modes at 495 and $518 \mathrm{~s}$ included.
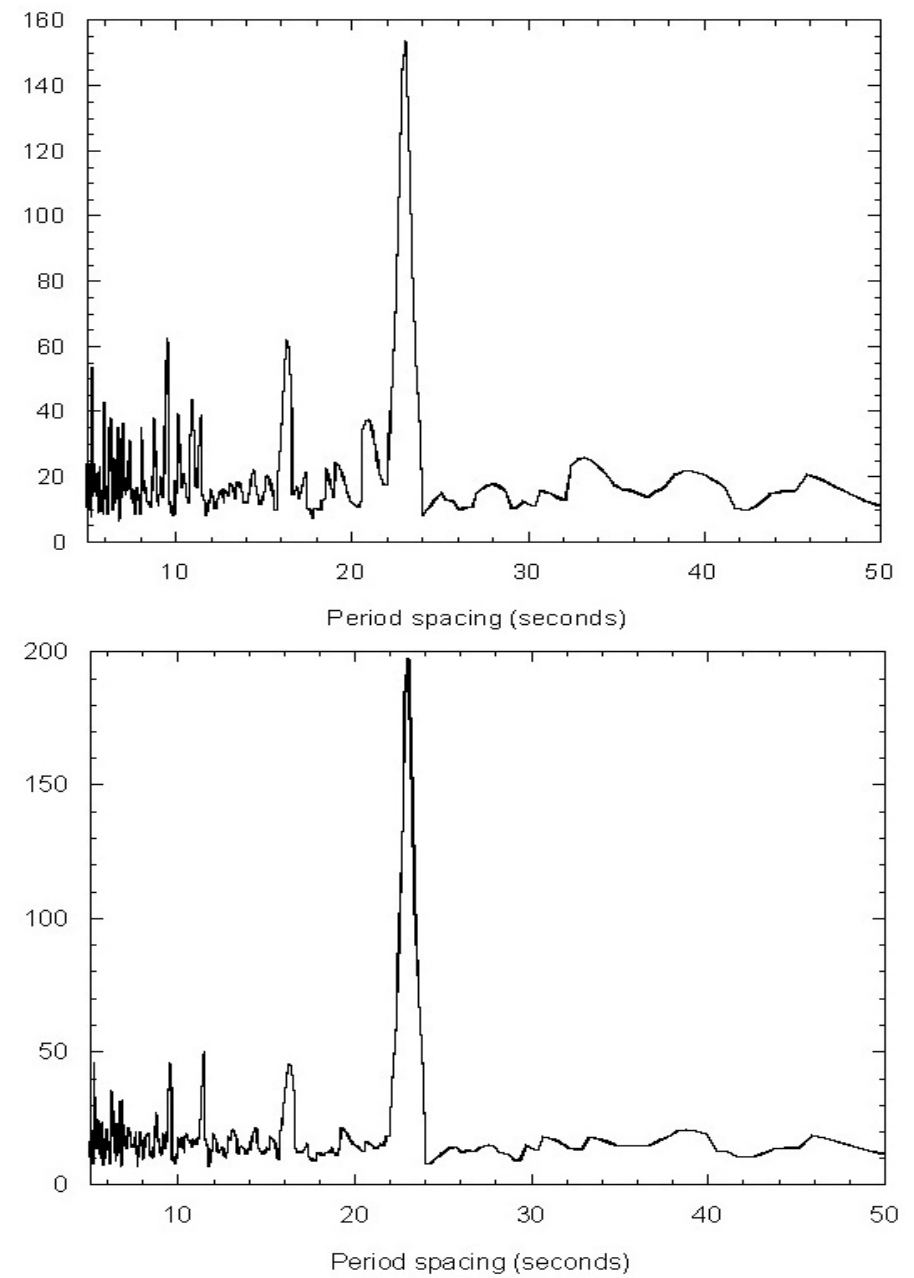

Fig. 11. Same as Fig. 9 from Inverse Variance test. Upper panel: the IV test of the seven $m=0$ mode periods. Lower panel: the same, but with the two single modes at 495 and $518 \mathrm{~s}$ included.

fixed at $10^{-3}$. We consider that the inferred $\Delta P$ is now reliable and can be used for a confident estimate of the stellar global parameters. Its value, $22.9 \mathrm{~s}$ is comparable to the period spacing derived for the other pre-white dwarf pulsators of PG 1159 type. In decreasing order of $T_{\text {eff }}$ these are: $21.62 \mathrm{~s}$ for RX J2117+3412 (Vauclair et al. 2002), $21.5 \mathrm{~s}$ for PG 1159-035 (Winget et al. 1991), $21.6 \mathrm{~s}$ for PG 2131+427 (Kawaler et al. 1995) and $23.0 \mathrm{~s}$ in PG 1707+427 (Kawaler et al. 2004).

Taking the period of the $m=0$ mode of the triplet which contains the largest amplitude mode as a reference period, one determines $\Delta k$, the number of orders separating this mode and the other modes detected in PG $0122+200 . \Delta k$ is listed in Table 5 together with the azimuthal number $m$ within each triplet.

\subsection{Mode trapping}

The fact that the period separations vary around the average period spacing of $22.9 \mathrm{~s}$ with a peak to peak amplitude of $\approx 5 \mathrm{~s}$ may be interpreted as the signature of mode trapping. Unfortunately, the number of missing modes makes difficult to estimate the value of the trapping cycle for PG $0122+200$. Knowing the average period spacing, we checked in our data, as well as in archive data, whether the missing modes predicted at periods close to $588 \mathrm{~s}, 542 \mathrm{~s}, 428 \mathrm{~s}$ and $359 \mathrm{~s}$ could be present below our detection limit. We did not find any evidence of peaks 


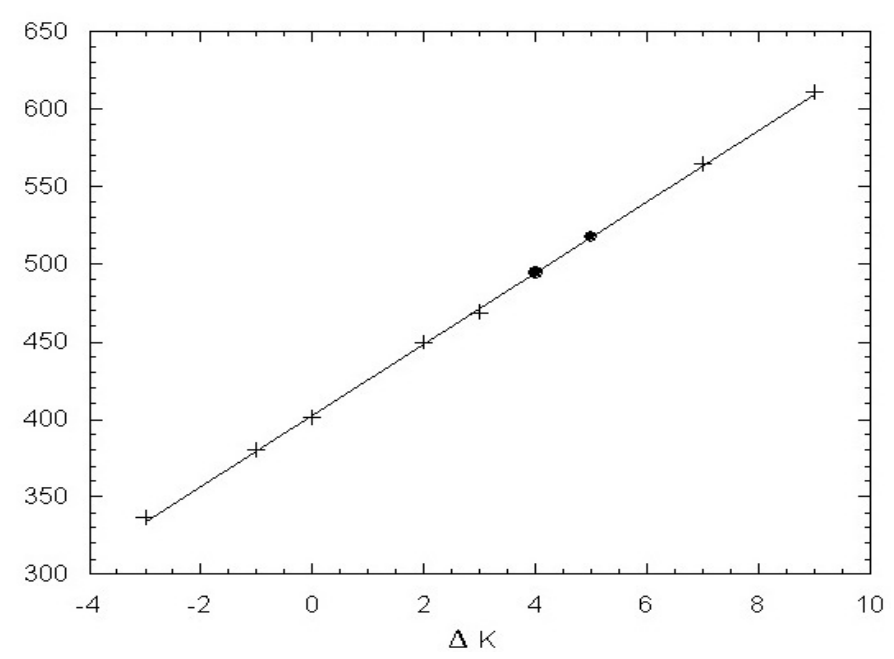

Fig. 12. Linear least-squares fit of the seven $m=0$ modes (crosses) as a function of $\Delta k$, the difference in radial order from the reference mode. The fit confirms a period spacing $\Delta P=22.9 \mathrm{~s}$. The two single modes at $517.96 \mathrm{~s}$ and $494.92 \mathrm{~s}$ (filled circles) fit nicely the period distribution with that value of the period spacing.

at the corresponding frequency with $S / N \geq 2 \times \sigma$. Using the available data, we nevertheless attempted to derive some information on the mode trapping. We compared the observed periods to the periods computed by using the average spacing of $22.9 \mathrm{~s}$ as derived by the linear least-squares fit shown in Fig. 12. Figure 13 shows the residuals of the observed period distribution relative to the average period spacing as a function of the period. It shows only one minimum centered on the mode at $449.5 \mathrm{~s}$ which should clearly be a trapped mode. There is a hint that the mode at $400 \mathrm{~s}$ could define a second minimum, or at least be close to it. Unfortunately, one did not detect the adjacent mode which should be at $\approx 428$ s so that we cannot claim that the $400 \mathrm{~s}$ mode is a trapped mode. We note, however, that if this mode was also a trapped mode, the trapping cycle period, defined as the period difference between adjacent trapped modes, would be around $68 \mathrm{~s}$, which corresponds to $\approx 3$ consecutive modes. This may be compared to the trapping cycle period of $83.9 \mathrm{~s}$ in RX J2117+3412 and of $80.6 \mathrm{~s}$ in PG 1159035 (Vauclair et al. 2002). From Kawaler \& Bradley's models (Kawaler \& Bradley 1994) it is known that the trapping cycle increases with decreasing helium-rich envelope mass fraction, $q_{y}$, at fixed luminosity (or $T_{\text {eff }}$ ) and decreases with decreasing $T_{\text {eff }}$ at fixed $q_{y}$. Combining the dependences of the trapping cycle on $q_{y}$ at constant luminosity on one hand and on $T_{\text {eff }}$ at constant $q_{y}$ on the other hand, Vauclair et al. (2002) derived an interpolation formula linking the helium-rich envelope mass fraction, $q_{y}$, the effective temperature and the trapping cycle, $T_{k}$, expressed in number of modes:

$\Delta \log \left(q_{y}\right)=16.93 \Delta \log \left(T_{\text {eff }}\right)-0.61 \Delta T_{k}$

where the symbol $\Delta$ represents the difference of a given parameter between two stars. Here we compare PG 0122+200 with PG 1159-035 whose parameters are $T_{\text {eff }}=140000 \mathrm{~K}$ (Dreizler $\&$ Heber 1998), $q_{y}=0.0035 M_{*}$ (Kawaler \& Bradley 1994) and $T_{k}=3.75$ (Vauclair et al. 2002). Taking the suggested trapping cycle of PG $0122+200$ at face value as $T_{k}=2.95$ we find that the helium-rich envelope mass fraction in PG $0122+200$ could be as low as $\log q_{y}=-6.0$. We should keep in mind, however, that this suggestion has to be considered with caution since it is not possible with the present data to prove that the mode at $400 \mathrm{~s}$ is a trapped mode. Would the next missing mode predicted at $428 \mathrm{~s}$ be the trapped mode instead, we get $\log q_{y}=-5.3$, still much less than in PG 1159-035.

If it is not possible to derive a definitive result on the helium-rich envelope mass fraction, we can make some interesting comparison with the hotter pre-white dwarf pulsators RX J2117+3412 and PG 1159-035 in which the mode trapping is unambiguously identified. The signature of the mode trapping in RX J2117+3412 is rather weak, with a semi-amplitude around the average period spacing for the $\ell=1$ modes of only $\approx 0.8 \mathrm{~s}$ (Vauclair et al. 2002). The variation in the period separations for the $\ell=1$ modes in PG $0122+200$ is much larger, with a peak to peak amplitude of $\approx 5 \mathrm{~s}$. This value is similar to the value of $\approx 6$ s observed in PG 1159-035 (Winget et al. 1991). This suggests that the region of strong $\mu$-gradient, which is due to the chemical transition between the outer He-C-N-O envelope and the $\mathrm{C}-\mathrm{O}$ interior, is closer to the surface and/or steeper in PG 0122+200 and PG 1159-035, than in RX J2117+3412. This is consistent with the following facts. Mass loss is observed during the hottest phases of the pre-white dwarf evolution, with a rate of $-7.4 \leq \log \dot{M} \leq-7.0$ for instance in RX J2117+3412 (Koesterke et al. 1998; Koesterke \& Werner 1998). The stars should have expelled a substantial fraction of their He-rich outer layers as they reach the luminosity of PG 1159-035 and a fortiori of PG $0122+200$, pushing the transition zone of strong $\mu$-gradient closer to the surface and making the mode trapping more efficient.

The $\mu$-gradient should also be steeper at more advanced phases of the evolution since the gravitational settling of the heavy elements increases the contrast in chemical composition between the regions above and below the transition zone. While the variation with decreasing $T_{\text {eff }}$ of the observed $C$ abundance in PG 1159 stars supports this argument, the observed $\mathrm{O}$ abundances do not provide any convincing constraint (Werner \& Herwig 2006).

One can also derive some more information from the mode trapping observed in GW Vir stars, compared to the one observed in RX J2117+3412. Most of the variation in the amplitude signature of the mode trapping appears between the $T_{\text {eff }}=170 \mathrm{kK}$ for RX J2117+3412 and the $T_{\text {eff }}=140 \mathrm{kK}$ for PG 1159-035. There is no significant further variation towards cooler $T_{\text {eff }}$ : the peak-to-peak amplitude in the $\Delta P$-vs.- $P$ diagrams are all around $5 \mathrm{~s}-6 \mathrm{~s}$ for PG 1159-035 ( $\approx 6 \mathrm{~s}$, Winget et al. 1991), PG 2131+066( $\approx 6 \mathrm{~s}$, Kawaler et al. 1995), PG 1707+427 ( $\approx 5.7 \mathrm{~s}$, Kawaler et al. 2004) and PG $0122+200(\approx 5.2 \mathrm{~s}$, this work). It suggests that the mass-loss efficiency decreases substantially below $140 \mathrm{kK}$. At cooler $T_{\text {eff }}$, further variation in the mode trapping amplitude (and cycle) should mainly result from the changes in the structure induced by gravitational settling. There may be a hint for such a decreasing amplitude, from PG 1159-035 to PG $0122+200$, indicating that the strong $\mu$-gradient zone is becoming deeper as the gravitational settling is processing after the mass loss has stopped. It will be necessary to also measure a trapping cycle in all the GW Vir stars to confirm this suggestion.

The results shown in Fig. 13 suggest some more comments. In considering the amplitudes of the five complete triplets detected in the 2002 data, we note that the largest amplitude mode at $400.4 \mathrm{~s}$ belongs to a probably trapped mode, as discussed above. However, there is no clear correlation between amplitude and trapping since the next two largest amplitude modes are untrapped. But we find that for the 3 largest amplitude modes, they all are the prograde component in their respective triplet, while for the next two lower amplitude modes the $m=0$ and the retrograde component respectively have the largest amplitude in their 


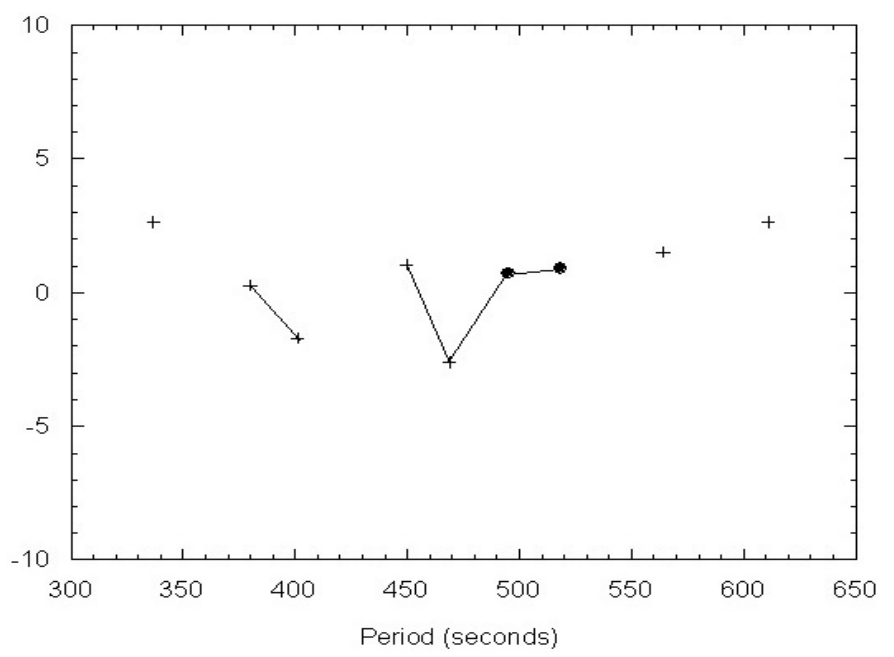

Fig. 13. A plot showing observational evidence of mode trapping. The residuals of the period distribution relative to the average period spacing $(\Delta P=22.9 \mathrm{~s})$ are plotted as a function of the period. The crosses represent the $m=0$ modes of the seven triplets. The filled circles represent the two single modes. Consecutive order modes are linked by straight lines. The modes defining minima on this plot are trapped modes while those defining maxima are nontrapped modes.

triplet. It suggests that there seems to be a correlation between the azimuthal order $m$ and the amplitude.

Finally, it is worth emphasizing that since the largest amplitude mode at $2497 \mu \mathrm{Hz}(400.4 \mathrm{~s})$ is a component of a probably trapped mode, or at least close to a trapped mode, its $\dot{P}$ measurement will provide information on the outer layers contaction timescale, but cannot be useful in determining the cooling timescale of PG $0122+200$. The next two largest amplitude modes at $2228 \mu \mathrm{Hz}(448.8 \mathrm{~s})$ and at $2973 \mu \mathrm{Hz}(336 \mathrm{~s})$ are untrapped modes and should be better indicators of the cooling timescale if one can derive their $\dot{P}$.

\subsection{The single modes}

The two single modes at $517.96 \mathrm{~s}$ and at $494.92 \mathrm{~s}$ fit the expected spacing for $\ell=1$ modes. They are two consecutive order $\ell=1$ modes. Relatively to the least-squares fit of the period distribution, as shown in Fig. 12, and adopting the average period spacing of $22.9 \mathrm{~s}$, we find that the expected $\Delta k=4$ and 5 modes fit the observed periods within $1.9 \mathrm{~s}$ and $1.7 \mathrm{~s}$ respectively. However, we cannot determine the $m$ value of these components since, at these periods, the rotational splitting induces components separated by only $\approx 1 \mathrm{~s}$ from the $m=0$ component and the signature of the rotational splitting may be hidden by the mode trapping.

\subsection{Mass and luminosity of PG $0122+200$}

The overall structure parameter $\Pi_{0}$ (see, e.g. Tassoul 1980) in pre-white dwarf pulsators is mainly related to the total mass of the stars. However, it also depends weakly on the luminosity and even more weakly on the He-rich envelope mass fraction $q_{y}$. For this reason one cannot disentangle the mass determination from the luminosity estimate, if one neglects the dependence on $q_{y}$. From a grid of evolutionary models covering the ranges of interest for these parameters, and their pulsation periods, Kawaler \& Bradley (1994) derived an approximate expression for $\Pi_{0}=\sqrt{\ell(\ell+1)} \times \Delta P_{\ell}$ as a function of the mass, the luminosity and $q_{y}$, where $\Delta P_{\ell}$ is the period spacing measured for the modes of degree $\ell$. We use this formula, corrected for a sign error in the power of the luminosity dependence (O'Brien 2000), to estimate the mass of PG $0122+200$ :

$\Pi_{0} \cong 15.5\left(M / M_{\odot}\right)^{-1.3}\left(L / 100 L_{\odot}\right)^{-0.035}\left(q_{y} / 10^{-3}\right)^{-0.00012}$.

Since $\Delta P_{\ell}$ has been estimated for the observed $\ell=1$ modes, we can derive the mass if we also know the luminosity and $q_{y}$. Due to the very weak dependence of $\Pi_{0}$ on $q_{y}$, the adopted value for the He-rich envelope mass fraction is not really important here. We choose a value $q_{y}=10^{-2}$. The luminosity of PG $0122+200$ is not known a priori. In the following discussion, we will choose a starting value of the luminosity to determine a first range of value for the mass, then derive a value for the radius from the surface gravity, from which we derive a new estimate for the luminosity. We repeat the process iteratively until it converges.

The interpolation formula of Kawaler \& Bradley (1994) has been obtained from models in the luminosity range $1.6 \leq$ $\log \left(L / L_{\odot}\right) \leq 3.0$. The previous estimate of PG $0122+200$ luminosity from O'Brien (2000), $\log \left(L / L_{\odot}\right)=0.7$, falls outside of this range. We use the interpolation formula nevertheless, to extrapolate the formula towards lower luminosity.

In this iterative procedure, we use the surface gravity, $\log g=$ $7.5 \pm 0.5$, from Dreizler \& Heber (1998). This large uncertainty on $\log g$ dominates the uncertainty on the derived luminosity. For this reason, we did not include in our estimate the uncertainty on $T_{\text {eff }}( \pm 4000 \mathrm{~K}$ according to Dreizler \& Heber 1998). The iterative process converges to a mass of $M / M_{\odot}=0.59 \pm 0.02$ and a luminosity $\log \left(L / L_{\odot}\right)=1.3 \pm 0.5$. The average value of the derived luminosity falls at the lower limit of the range of luminosities that Kawaler \& Bradley (1994) considered to obtain their approximate formula. This legitimates our procedure.

The asteroseismologic mass of $0.59 \pm 0.02 M_{\odot}$ falls within the range of masses $0.53 \pm 0.1 M_{\odot}$ determined from spectroscopy by Dreizler \& Heber (1998). Its accuracy is however improved by a factor of 5. The mass of PG $0122+200$ is consequently strongly contrained by asteroseismology. The value of $0.58 M_{\odot}$ that Werner \& Herwig (2006) derive from a comparison with the evolutionary tracks of Schönberner (1983) is in better agreement with the asteroseismic value. However, the new evolutionary tracks by Miller Bertolami et al. (2006) puts the PG 0122+200 mass back to the Dreizler \& Heber's (1998) value. This is difficult to reconcile with the asteroseismologic value derived from Kawaler \& Bradley's (1994) models. In the case of RX J2117+3412 that Corsico et al. (2007) have reanalysed using the evolutionary tracks by Miller Bertolami et al. (2006) the derived mass $\left(0.565 M_{\odot}\right)$ is in excellent agreement with the mass of $0.56 M_{\odot}$ derived by Vauclair et al. (2002) from the Kawaler \& Bradley's (1994) models.

\subsection{Distance}

Having determined an approximate value of the luminosity, one can estimate the distance of PG $0122+200$. We adopt a bolometric correction of $-6.0 \pm 0.5$ from Werner et al. (1991). Incidently, this value is identical to the value determined for hot DA white dwarfs by Bergeron et al. (1995) which is -6.1 for $T_{\text {eff }}=80 \mathrm{kK}$. Using our own estimate of the $V$ magnitude $V=16.8 \pm 0.1$ and taking into account the uncertainty on both the luminosity and the bolometric correction, we derive a distance:

$D \cong 0.7_{-0.4}^{+1.0} \mathrm{kpc}$

We predict an upper limit on the parallax of 3.2 mas. 
Due to the large uncertainty on the luminosity, mainly due to the poorly determined surface gravity, the constraint on the distance is not strong. Inversely, a precise measurement of the parallax would improve the luminosity and surface gravity estimates since the mass is strongly constrained by the asteroseismology.

\subsection{The neutrino luminosity of $P G 0122+200$}

One of the main interests in studying PG $0122+200$ is that it is predicted from theory to cool predominantly through neutrino emission (O'Brien et al. 1998; O'Brien \& Kawaler 2000). Earlier estimates, based on a mass of PG $0122+200$ as high as $0.66 M_{\odot}$ predicted a ratio of neutrino luminosity on photon luminosity of about 2.5 at the effective temperature of PG $0122+200$. With the reduced total mass derived in the present paper, this ratio decreases accordingly. Compared to the predictions for a $0.60 M_{\odot}$ model of pre-white dwarf (O'Brien \& Kawaler 2000) the ratio is $1.6 \pm 0.2$ in the case where the neutrino luminosity is estimated by using the normal rate from Ito et al. (1996) (see O'Brien \& Kawaler 2000 for details). The quoted uncertainty includes only the uncertainty on the effective temperature of PG $0122+200$. The ratio decreases to $1.2 \pm 0.2$ for the rates diminished by a factor of 3 , and increases to $2.0 \pm 0.2$ for a rate enhanced by a factor 3. So, even with a somewhat reduced mass, PG $0122+200$ is still a good candidate to test the physics of neutrino production in dense plasma. The ratio between the two main processes of neutrino production, the plasmon and the bremsstrahlung neutrino processes, is unsensitive to the adopted rate at the temperature of PG $0122+200$. For the $0.6 M_{\odot}$ model, the luminosity predicted at the temperature of PG $0122+200$ is $\approx \log \left(L / L_{\odot}\right)=1.0$. The luminosity derived from the present asteroseismic study, $\log \left(L / L_{\odot}\right)=1.3 \pm 0.5$ is in good agreement with the theoretical predictions. However, its uncertainty is too large to provide any useful constraint on the correct rate of neutrino production. This large uncertainty comes from the poor spectroscopic derivation of the surface gravity. According to O'Brien \& Kawaler (2000), modifying the rate of neutrino production by a factor 3 , compared to the normal rate of Ito et al. (1996), only changes the luminosity by $\approx 0.1 \mathrm{dex}$ for the mass and the temperature of PG $0122+200$. To discriminate between the different rates of neutrino production from PG $0122+200$ observations, it would be necessary to improve the surface gravity measurement by at least one order of magnitude. This is probably a long way from the present uncertainty of $0.5 \mathrm{dex}$. A precise parallax measurement would better constrain the surface gravity. Another useful constraint on the neutrino rate should come from the measurement of $\dot{P}$ for one or more untrapped modes.

\section{Conclusions}

From a set of new photometric multi-site campaigns on the PG 1159 pulsator PG $0122+200$, we have identified 23 frequencies, distributed in 7 triplets and 2 single frequencies. We do not see any linear combinations of frequencies and we conclude that all the observed frequencies correspond to real stellar modes. The frequency distribution is remarquably stable since the discovery data which indicates that a $\dot{P}$ measurement is possible for PG $0122+200$. Since all the observed multiplets are triplets, we interpret them as $\ell=1$ modes split by rotation. We derived an average rotational splitting of $3.74 \mu \mathrm{Hz}$ which translates into a period of rotation of 1.55 days. The observed periods range from $612 \mathrm{~s}$ to $336 \mathrm{~s}$ and are all compatible with $\ell=1$ modes. We find a correlation between the azimuthal order $m$ and the amplitude, the largest amplitude modes being systematically the prograde components in their respective triplets. We update the value of the average period spacing, $\Delta P=22.9 \mathrm{~s}$. We identify a signature of mode trapping but the missing modes do not allow us to determine precisely the He-rich envelope mass fraction. However, we are able to identify trapped and untrapped modes. We show that the largest amplitude mode is probably a trapped mode, i.e. not suitable for the estimate of the cooling time scale from its $\dot{P}$ measurement. We infer that the helium-rich outer layers mass fraction in PG $0122+200$ seems to be much lower than in PG 1159-035, with $-6.0 \leq \log \left(q_{y}\right) \leq-5.3$. The amplitude of the mode trapping suggests that the transition zone of large $\mu$-gradient responsible for the mode trapping must be closer to the stellar surface in PG 0122+200 and in PG 1159-035 than in the hotter PG 1159 star RX J2117+3412. This is in agreement with the fact that between the evolutionary stages corresponding to RX J2117+3412 and the cooler stars PG $1159-035$ and PG $0122+200$, a substantial fraction of the He-rich outer layers have been eroded by mass-loss. From the period spacing, we derive a mass of $0.59 \pm 0.02 M_{\odot}$, in excellent agreement with the spectroscopically determined mass of $0.58 M_{\odot}$ derived by Werner \& Herwig (2006) but in poorer agreement with the $0.53 M_{\odot}$ derived by Dreizler \& Heber (1998) and Miller Bertolami et al. (2006). From the asteroseismological mass, we evaluate the luminosity and the distance of PG $0122+200: \log L / L_{\odot}=1.3 \pm 0.5$, $D \cong 0.7_{-0.4}^{+1.0} \mathrm{kpc}$. Both the luminosity and the distance cannot be better constrained due to the large uncertainty on the spectroscopically determined surface gravity. From the marginally weak asymmetry observed within the components of the triplets, we derive a possible limit to the strength of the magnetic field: $B \leq 4 \times 10^{3}$ G. Finally, we revise the predicted neutrino luminosity and conclude that PG $0122+200$ is still a good candidate to check the value of the still uncertain neutrino emission rate. To reach that goal, a $\dot{P}$ measurement for the untrapped modes is underway.

Acknowledgements. We thank an anonymous referee whose comments helped us improve a first version of the manuscript. J.-N. F. acknowledges support from the National Natural Science Foundation of China, throught the Grant 10543001, and from Beijing Normal University, through the Grant 107021. G.V. and M.C. acknowledge support from the exchange programme between the Centre National de la Recherche Scientifique and the Academy of Sciences of China. Part of the data presented here have been taken using ALFOSC, which is owned by the Instituto de Astrofisica de Andalucia (IAA) and operated at the Nordic Optical Telescope under agreement between IAA and the NBIfAFG of the Astronomical Observatory of Copenhagen. M.W. acknowledges support from NSF grant AST0205902. S.D. and S.S. acknowledge support from the /Deutsche Forschungsgemeinschaft/ under travel grants DR 281/16-1. Part of the work is based on observations collected at the German-Spanish Astronomical Center, Calar Alto, operated by the Max-Planck-Institut für Astronomie jointly with the Spanish National Commission for Astronomy.

\section{References}

Bergeron, P., Wesemael, F., \& Beauchamp, A. 1995, PASP, 107, 1047 Bond, H., \& Grauer, A. 1987, ApJ, 321, L123

Bond, H., Kawaler, S. D., Ciardullo, R. et al. 1996, AJ, 112, 2699

Brickhill, A. J. 1975, MNRAS, 170, 404

Corsico, A. H., Althaus, L. G., Miller Bertolami, M. M., \& Werner, K. 2007, A\&A, 461, 1095

Dreizler, S., \& Heber, U. 1998, A\&A, 334, 618

Handler G., Pikall H., O'Donoghue D. et al., 1997, MNRAS 286, 303

Ito, N., Hayashi, H., Nishikawa, A., \& Kohyama, Y. 1996, ApJS, 102, 411

Jones, P. W., Pesnell, W. D., Hansen, C. J., \& Kawaler, S. D. 1989, ApJ, 336, 
403

Kawaler, S. D. 1988, in Advances of Helio- and Asteroseismology, ed. J. Christensen-Dalsgaard \& S. Frandsen (Dordrecht: Reidel), 329

Kawaler, S. D., \& Bradley, P. A. 1994, ApJ, 427, 415

Kawaler, S. D., O’ Brien, M. S., Clemens, J. C. et al. 1995, ApJ, 450, 350

Kawaler, S. D.,Potter, E. M., Vuckovic, M. et al. 2004, A\&A, 428, 969

Koesterke, L., \& Werner, K. 1998, ApJ, 500, L55

Koesterke, L., Dreizler, S., \& Rauch, T. 1998, A\&A, 330, 1041

Lenz, P., \& Breger, M. 2005, Comm. in Asteroseismology, 146, 53

Miller Bertolami, M. M., Althaus, L. G. 2006, A\&A, 454, 845

Nather, R. E., Winget, D. E., Clemens, J. C., Hansen, C. J., \& Hine, B. P. 1990, ApJ, 361, 309

O’Brien, M. S., \& Kawaler, S. D. 2000, ApJ, 539, 372

O’Brien, M. S., Clemens, J. C., Kawaler, S. D., \& Dehner, B. T. 1996, ApJ, 467, 397

O’ Brien, M. S., Vauclair, G., Kawaler, S. D. et al. 1998, ApJ, 495, 458
O’Donoghue, D. 1994, MNRAS, 270, 222

Rauch, T., \& Werner, K. 1997, The Third Conference on Faint Blue Stars, ed. A. G. D. Philip, J. Liebert \& R. A. Saffer (Schenectady, NY: L. Davis Press), 217

Reed, M. D., Kawaler, S. D., \& O’Brien, M. S. 2000, ApJ, 545, 429

Scargle, J. D. 1982, ApJ, 263, 835

Schönberner, D. 1983, ApJ, 272, 708

Tassoul, M. 1980, ApJS, 43, 469

Vauclair, G., Pfeiffer, B., Grauer, A., Belmonte, J.-A. et al. 1995, A\&A, 299, 707

Vauclair, G., O’Brien, M. S., Fu, J.-N. et al. 2001, in 12th European Workshop on White Dwarf Stars, ed. J. L. Provencal, H. L. Shipman, J. MacDonald \& S. Goodchild, ASP Conf. Ser., 226, 293

Vauclair, G., Moskalik, P., Pfeiffer, B. et al. 2002, A\&A, 381, 122

Werner, K., \& Herwig, F. 2006, PASP, 118, 183

Werner, K., Heber, U., \& Hunger, K. 1991, A\&A, 244, 437

Winget, D. E., Nather, R. E., Clemens, J. C. et al. 1991, ApJ, 378, 326 